Arab Journal of Nuclear Sciences and Applications

Web site: ajnsa.journals.ekb.eg

ISSN 1110-0451

\title{
Relations Between Radionuclides Activities and Pb Concentration on Different Rock Types
}

\author{
E. S. Abd EL-Halim ${ }^{1}$ and Asma Mohammed AL-abrdi ${ }^{2}$ \\ (1) Faculty of Women for Arts, Science and Education. Physics Department, Ain Shams University, Egypt. \\ (2) physics department, College of science Omar AL-mokhtar University, Libya.
}

\begin{tabular}{l} 
ART ICLE INFO \\
\hline Article history: \\
Received: $30^{\text {th }}$ Nov. 2020 \\
Accepted: $1^{\text {st }}$ Apr. 2021 \\
\hline Keywords: \\
Natural radioactivity, \\
HPGe detector, \\
Sediment rocks, \\
Granite; Leaching.
\end{tabular}

\begin{abstract}
The level of natural radioactivity in eight sediment rock samples collected from western south Sinai from three different locations Um Bogma, Wadi Um Hamd and wadi Sad El-Banat, were measured. Also, two granite samples, collected from Gabal Gattar, north eastern desert in Egypt was investigated. The gamma-ray spectrometry system with hyper pure germanium (HPGe) detector was been used to determined radioactivity concentrations. Samples were subjected to sulphuric acid leaching processes with the same parameters of solid liquid ratio $\mathrm{S} / \mathrm{L}$, acid concentration and leaching time. After the leaching process, the pregnant solution was separated from the residual and the latter was dried. The two units, named the pregnant solutions and residuals were also measured radiometrically using the HPGe detector to determine the activity concentrations $(\mathrm{Bq} / \mathrm{kg})$ of the different radionuclides of samples.
\end{abstract}

The results showed that the relation between the sum of activities of ${ }^{238} U$ in both solutions and residuals with originals have two modes. The first mode is represented by the sum of ${ }^{238} \mathrm{U}$ activities in leachate and residual is nearly equal the activity in the original sample. While the second mode, the sum is higher than the original.

These variations depend mainly on the grain surfaces in the different rock types and its content high $\mathrm{Pb}$ concentration. This phenomenon varied in magnitude within the different radionuclides in each sample. Also, the leachability of $U$ by the chemical analysis using several acidic leaching experiments for uranium have been performed using sulfuric acid.

\section{INTRODUCTION}

Natural radionuclides are present in all rock materials in varying amounts depending on their concentration levels in sources rock materials. The three naturally occurring radioactive decay chains include the ${ }^{238} \mathrm{U},{ }^{235} \mathrm{U}$ and ${ }^{232} \mathrm{Th}$, which decay through a series of radioactive elements up to stable $\mathrm{Pb}$ isotopes. These series are the main sources of gamma radiation in rocks, soils and water. Gamma ray from these radioisotopes represents one of the main external sources of population exposure [1]. The knowledge of distribution of the primordial radionuclides is an important role for peoples avoid long exposure.

In the leaching process, oxidation potential, temperature, and $\mathrm{pH}$ of the solution are important parameters, and are often manipulated to optimize dissolution of the desired metal component into the aqueous phase [2]. The ${ }^{226} \mathrm{Ra}$ is the main radionuclide in the tailings after leaching process for uranium extraction and is highly active with its solid decay products. The main danger is not only during the radioactive releases during leaching processes, but also for many years after cessation of operations [3]. Sulfuric acid as an acidic reagent is widely used for uranium leaching because of its availability and its low cost [4]. The physical and chemical processes used to extract uranium from ore, such as crushing and acid treatment produce large amounts of mill tailings. Studies of the behavior of most of different radionuclides in the ${ }^{238} \mathrm{U}$ and ${ }^{232} \mathrm{Th}$ series during the acidic leaching concluded that the nuclides before ${ }^{226} \mathrm{Ra}$ in the ${ }^{238} \mathrm{U}$ decay series are easily released in the pregnant solution than the ${ }^{226} \mathrm{Ra}$ itself and its solid daughters $\left({ }^{214} \mathrm{~Pb}\right.$ and $\left.{ }^{214} \mathrm{Bi}\right)[5][6]$. 


\section{Sample Description and Preparation:}

Sediment samples were collected from south western Sinai, Egypt, four samples from middle member of Um Bogma formation, $2 \mathrm{~S}$ shale, $3 \mathrm{~S}$ siltstone, $7 \mathrm{~S}$ claystone and $17 \mathrm{~S}$ Gibbsite-bearing shale. Two samples from wadi Um Hamd (A1, A2), two samples from wadi Sad ElBanat (A3, A4) and two granite samples (2G, 5G) were collected from Gabal Gattar, north eastern desert. The samples were collected from a depth of $10 \mathrm{~cm}$. Each sample was collected from an area corresponding to one $\mathrm{m}^{2}$ and was homogenized in situ, and this sand mixture, weighing approximately one $\mathrm{kg}$.

The collected samples were prepared for $\gamma$-ray spectrometric analysis by HPGe detector. The samples were first dried, crushed and sieved to about 200 meshes. The samples were placed in polyethylene bottles and weighted. The bottles were completely sealed for one month to allow radioactive equilibrium to be reached. This step is necessary to ensure that radon gas is confined within the volume and that the daughters will also remain in the sample.

\section{Experimental Technique}

\section{Leaching Experiments}

The leaching process was carried out by using $\mathrm{H}_{2} \mathrm{SO}_{4}$, acid (150 g sediments and granite sample weight under the conditions; solid/liquid ratio 1:3, acid concentration $30 \%$, stirring time one hour and at room temperature). Filtration was carried out to separate the leachate from residual which is dried. The leachate was packed well in $250 \mathrm{ml}$ marinelli and also the residual was left for one month for measurements by HPGe. High purity vertical germanium was coupled to a PCcomputer with a special electronic card to make it equivalent to a multichannel analyzer. The system also contains the usual electronic components of preamplifier, amplifier and power supply. The detector has resolution (FWHM) of $1.85 \mathrm{keV}$ for the $1332.5 \mathrm{keV}$ $\gamma$-ray line of ${ }^{60} \mathrm{Co}$. The $\gamma$-ray spectrometer energy calibration was performed using ${ }^{60} \mathrm{Co},{ }^{226} \mathrm{Ra}$ and ${ }^{241} \mathrm{Am}$ point sources. The detector was surrounded by a special heavy lead shield of $10 \mathrm{~cm}$ thickness with inside dimension $28 \mathrm{~cm}$ diameter $40.5 \mathrm{~cm}$ height. To achieve a high efficiency of the detection, it is indispensable to assure that the highest possible number of particles emitted from the source enters into the effective volume of the detector. This can be achieved by the choice of the maximum possible measurement geometry, by exclusion Sample itself (self-absorption) and by exclusion or limitation of the kinetic energy losses of the detected particles between the sample and the effective volume of the detector [6]. For efficiency calibration, it is essential that nuclides are used for which the gamma ray emission probabilities are known accurately and that a source of known activity is used. Whenever possible, sources that have been certified as to their radioactive content should be used [7]. The absolute detection efficiency of the HPGe detector was determined by using three wellknown reference materials obtained from the International Atomic Energy Agency for $\mathrm{U}$, Th and $\mathrm{K}$ activity measurements: RGU-1, RGTh-1 and RGK-1 [8] [9]. The sample containers were placed on top of the detector for counting. The same geometry and size were used for both the samples and the reference materials [10]. The uranium reference material (RGU-1) is U-ore diluted with silica with $4940 \mathrm{~Bq} \cdot \mathrm{Kg}^{-1}$ of ${ }^{238} \mathrm{U}, 228$ Bq. $\mathrm{Kg}^{-1}$ of ${ }^{235} \mathrm{U}$, a negligible amount of ${ }^{40} \mathrm{~K}$ ( less than $0.63 \mathrm{~Bq} \cdot \mathrm{Kg}^{-1}$ ) and some traces of ${ }^{232} \mathrm{Th}$ (less than 4 Bq. $\mathrm{Kg}^{-1}$ ). The thorium reference material (RGTh-1) is Th-ore diluted with silica having $3250 \mathrm{~Bq} \cdot \mathrm{Kg}^{-1}$ of ${ }^{232} \mathrm{Th}$, but containing some ${ }^{238} \mathrm{U}\left(78 \mathrm{~Bq} \cdot \mathrm{Kg}^{-1}\right)$ and ${ }^{40} \mathrm{~K}(6.3$ Bq. $\left.\mathrm{Kg}^{-1}\right)$. The potassium calibration reference material (RGK-1) is produced from high purity (99.8\%) potassium sulphate with $14000 \mathrm{~Bq} \cdot \mathrm{Kg}^{-1}$ of potassium with uranium and thorium contents lower than 0.001 and $0.01 \mathrm{ppm}$, respectively [9]. Uranium-238 activity was determined indirectly from the gamma rays emitted by its daughter products $\left({ }^{234} \mathrm{Th}\right.$ and ${ }^{234 \mathrm{~m}} \mathrm{~Pa}$ ) whose activities are determined from the $63.3(3.9 \%)$ and 1001(0.7\%) $\mathrm{keV}$ photopeaks, respectively.

The gamma-ray transitions of ${ }^{228} \mathrm{Ac}$ (338.4 (12.3\%), $911(29 \%)) \mathrm{KeV},{ }^{212} \mathrm{Bi}(727.3 \mathrm{KeV}(7 \%))$ and ${ }^{208} \mathrm{Tl}$ $(583.1 \mathrm{KeV}(30 \%))$ were used to evaluate the specific activity of ${ }^{232} \mathrm{Th}$. The activity concentration of ${ }^{226} \mathrm{Ra}$ was measured from $186.1 \mathrm{KeV}(3.29 \%) .{ }^{40} \mathrm{~K}$ was determined directly from the $1460 \mathrm{KeV}(10.7 \%)$ peak energy [11].

The background contribution due to naturally occurring radionuclides in the environment around the detector is determined; an empty polyethylene beaker of the same $250 \mathrm{~cm}^{3}$ volume was counted with the same geometrical conditions as the sample. The measurement time for both activity and background measurement was 70000 seconds. The background spectra were used to correct the net- gamma- ray peak areas for the studied isotopes.

The Leachability of the radionuclides was calculated according to the following equation:

$$
\text { Leachability }(\%)=\frac{\text { Activity concentration in leachate }}{\text { Activity concentration in original }} \times 100
$$




\section{X - Ray fluorescence (XRF) Spectrometry}

XRF was used to determine the chemical analysis of major and trace elements. In this study, the concentration of trace elements was determined to focus light from the presence of high concentration for lead in most samples.

In this study, the trace elements is measured at the laboratories of the National Research Center (NRC), Egypt, by the X-ray fluorescence technique (XRF) using Philips Unique II unit fitted with an sample changer PW 1510 (30 position). The instrument is connected to a computer system using X-40 program for spectrometry. The detection limit of the measured elements by XRF technique was estimated at $2 \mathrm{ppm}$ for $\mathrm{Rb}, \mathrm{Nb}, \mathrm{Ga}, \mathrm{Y}$ and $\mathrm{S}$, at $8 \mathrm{ppm}$ for $\mathrm{pb}$ and $\mathrm{Cu}$ and $5 \mathrm{ppm}$ for other measured trace elements [12].

\section{RESULTS AND DISCUSSION}

\section{1-Geochimical analyses:}

The concentration of the trace elements in (ppm) for the samples under investigation is shown in figure (1) which represent high concentration in $\mathrm{Ni}, \mathrm{Zn}, \mathrm{V}$ and $\mathrm{Co}$, samples $2 \mathrm{~S}$ and $17 \mathrm{~S}$ show high concentration in $\mathrm{Pb}$ (1065 and $4088.96 \mathrm{ppm}$ ), respectively. Samples A1and A4 has high concentration of $\mathrm{Zn}, \mathrm{Sr}, \mathrm{Zr}, \mathrm{Ba}, \mathrm{Pb}, \mathrm{U}, \mathrm{V}$ and $\mathrm{Cr}$. While the samples $2 \mathrm{G}$ and $5 \mathrm{G}$ have high concentration in $\mathrm{Rb}, \mathrm{Zr}, \mathrm{Cr}$.
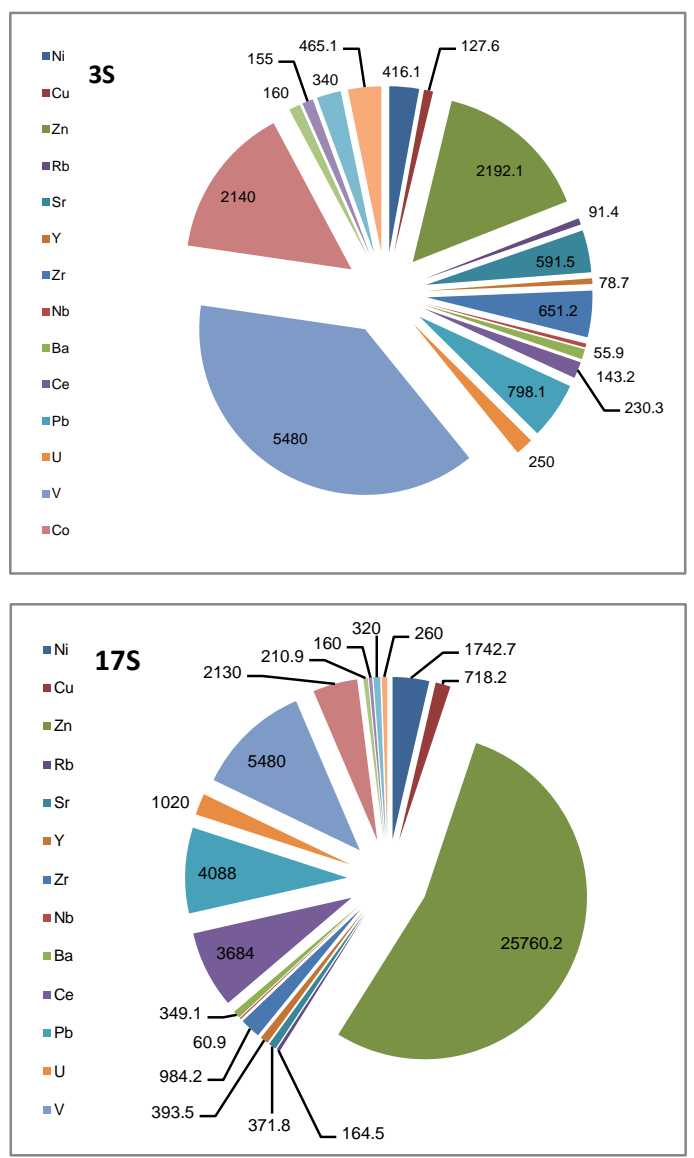

Arab J. Nucl. Sci. Appl., Vol. 54, 3, (2021)
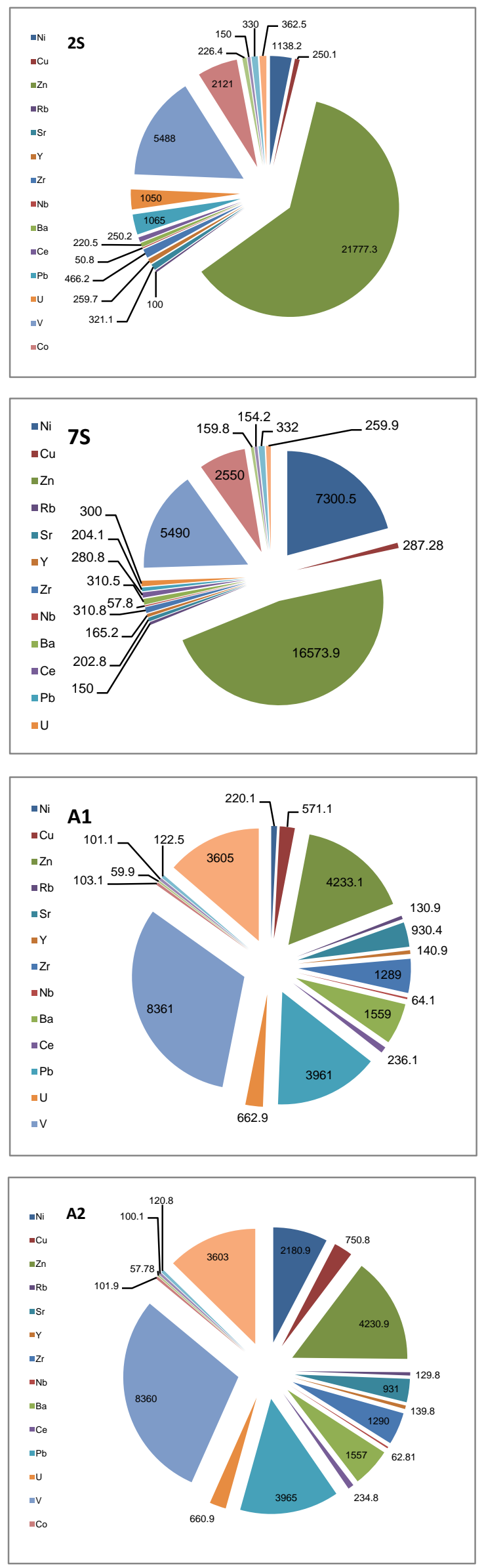

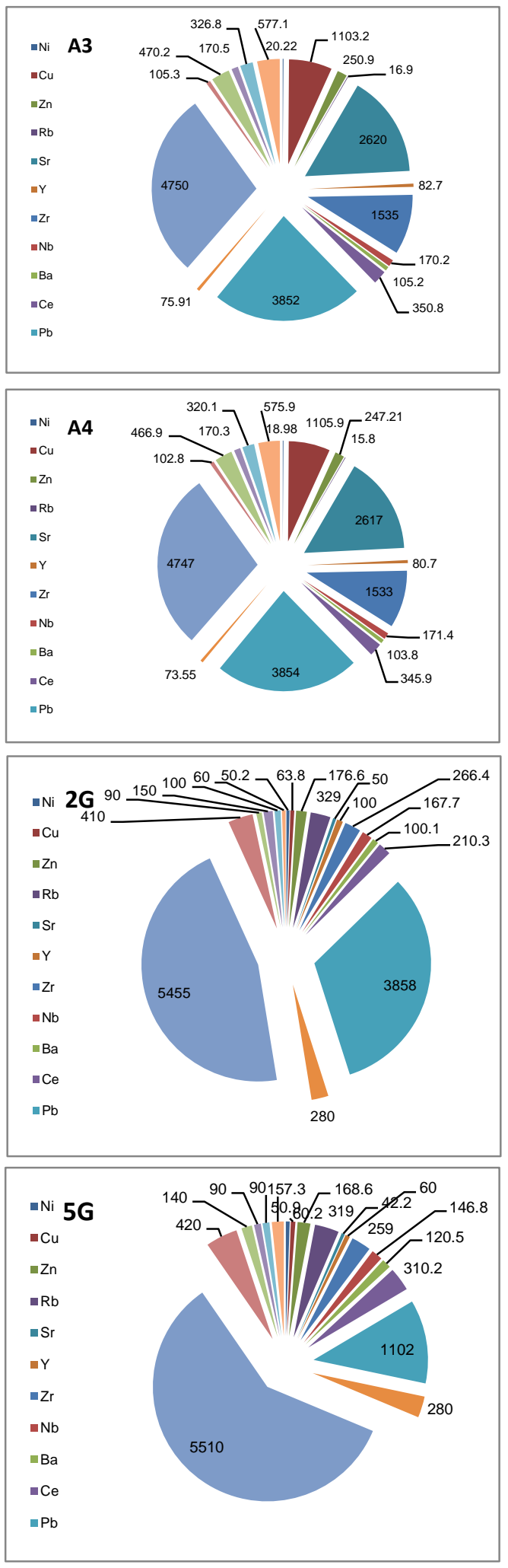

Fig. (1): Trace elements content (ppm) in the studied samples

\section{2- Radiometric Measurements}

The distribution of radionuclides during samples processing includes, distributions in original samples, leachate (pregnant solution) and residual as shown in Tables (1-10), which are analyzed by gamma ray- detector. This difference in gamma activity is sometimes +ve (the sum is greater than the original) and in other time-ve (the sum is lower than the original). This difference is varied mostly with the variation of geochemical composition of the rock samples.

The activity concentration of ${ }^{238} \mathrm{U}$ in the original samples, residuals and pregnant solutions are higher than the typical world average value of $33 \mathrm{~Bq} / \mathrm{kg}$ [13].

The behavior of each radionuclide in the samples shows that the ${ }^{226} \mathrm{Ra}$ Activity concentration is higher in the residual than that in the original for all samples. Also, the activity concentration of ${ }^{226} \mathrm{Ra}$ in the original, residual and solution are higher than the typical world average value of $32 \mathrm{~Bq} / \mathrm{kg}$ [13].

For ${ }^{232} \mathrm{Th}$ all the original samples are higher than the permissible value of $45 \mathrm{~Bq} / \mathrm{kg}$. Also, the residuals samples are higher than the permissible level 45 except sample $17 \mathrm{~S}$. For ${ }^{40} \mathrm{~K}$ the original samples are higher than the permissible level $412 \mathrm{~Bq} / \mathrm{kg}$ except samples $2 \mathrm{~S}, 7 \mathrm{~S}, \mathrm{~A} 3$ and A4, while the residual samples are higher than the original in all samples [13].

The summation of the activities of the measured radionuclides of ${ }^{238} \mathrm{U}$ in solution and residual is less than original as shown in samples $3 \mathrm{~S}$ and7S. From figure (1), it can be concluded that these samples contain low concentration of $\mathrm{Pb}$ (798.08 and $204.16 \mathrm{ppm}$ ) respectively. While the summation of the activities of the measured radionuclides of ${ }^{238} \mathrm{U}$ in samples $2 \mathrm{~S}, 17 \mathrm{~S}, \mathrm{~A} 1, \mathrm{~A} 2, \mathrm{~A} 3, \mathrm{~A} 4$, $2 \mathrm{G}$ and $5 \mathrm{G}$ are higher than the original. In these samples the lead $(\mathrm{Pb})$ content is 1065, 4088.96, 3961.13, 3965.91, $3852.123854 .85,3858.36$ and 1102.08 ppm respectively.

Table (1): Activity concentrations of radionuclides in original samples, residuals and leachates (pregnant solutions) for sample No. 7S (claystone).

\begin{tabular}{|c|c|c|c|c|c|}
\hline Radionuclide & $\begin{array}{l}\text { Original } \\
(\mathrm{Bq} / \mathrm{Kg})\end{array}$ & $\begin{array}{c}\text { Solution } \\
\text { (Bq/l) }\end{array}$ & $\begin{array}{c}\text { Leachability } \\
(\%)\end{array}$ & $\begin{array}{l}\text { Residual } \\
(\mathbf{B q} / \mathbf{K g})\end{array}$ & $\begin{array}{c}\text { Summation } \\
\text { (Residual+Solution)/ } \\
\text { Original }(\%)\end{array}$ \\
\hline \multicolumn{6}{|l|}{${ }^{238}$ Useries } \\
\hline${ }^{234} \mathrm{Th}$ & 2941.5 & 865.9 & 29.437 & 3097.4 & 134.737 \\
\hline${ }^{234 \mathrm{~m}} \mathrm{~Pa}$ & 3318.5 & 518.3 & 15.618 & 3045.2 & 107.382 \\
\hline Average & 3130.0 & 692.15 & 22.113 & 3071.3 & 120.238 \\
\hline${ }^{234} \mathrm{U}$ & 333.2 & 390.95 & 11.728 & 1124.3 & 45.459 \\
\hline${ }^{230} \mathrm{Th}$ & 2264.7 & 1330.55 & 5.897 & 1565.7 & 75.032 \\
\hline \multicolumn{6}{|l|}{$\begin{array}{c}{ }^{226} \mathrm{Ra} \\
\text { subseries }\end{array}$} \\
\hline${ }^{226} \mathrm{Ra}$ & 2448.7 & 62.96 & 2.5711 & 3230.8 & 134.510 \\
\hline${ }^{214} \mathrm{~Pb}$ & 2081.9 & 19.18 & 0.921 & 2164.21 & 104.874 \\
\hline${ }^{214} \mathrm{Bi}$ & 1983.2 & 20.61 & 1.039 & 1898.1 & 96.748 \\
\hline${ }^{235} \mathrm{U}$ & 155.8 & 34.5 & 22.143 & 70.95 & 67.682 \\
\hline \multicolumn{6}{|l|}{${ }^{232} \mathrm{Th}$ series } \\
\hline${ }^{228} \mathrm{Ac}$ & 59.2 & 8.53 & 14.408 & 54.17 & 105.912 \\
\hline${ }^{208} \mathrm{Tl}$ & 59.14 & 7.51 & 12.698 & 45.12 & 88.992 \\
\hline Average & 59.19 & 8.02 & 13.549 & 49.64 & 97.415 \\
\hline${ }^{40} \mathrm{~K}$ & 352.5 & 106.6 & 30.241 & 609.46 & 203.137 \\
\hline
\end{tabular}


Table (2): Activity concentrations of radionuclides in original samples, residuals and leachates (pregnant solutions) for sample No. 17S (Gibbsite).

\begin{tabular}{|c|c|c|c|c|c|}
\hline Radionuclide & $\begin{array}{l}\text { Original } \\
(\mathrm{Bq} / \mathrm{Kg})\end{array}$ & $\begin{array}{c}\text { Solution } \\
\text { (Bq/l) }\end{array}$ & $\begin{array}{c}\text { Leachability } \\
(\%)\end{array}$ & $\begin{array}{l}\text { Residual } \\
(\mathrm{Bq} / \mathrm{Kg})\end{array}$ & $\begin{array}{c}\text { Summation } \\
\text { (Residual+Solution) } / \\
\text { Original }(\%)\end{array}$ \\
\hline \multicolumn{6}{|l|}{${ }^{238}$ Useries } \\
\hline${ }^{234} \mathrm{Th}$ & 3724.3 & 1212.32 & 32.551 & 3862.79 & 136.270 \\
\hline${ }^{234 \mathrm{~m}} \mathrm{~Pa}$ & 4103.02 & 1304.78 & 31.860 & 4907.85 & 151.416 \\
\hline Average & 4103.02 & 1258.55 & 30.673 & 4385.33 & 137.554 \\
\hline${ }^{234} \mathrm{U}$ & 4198.98 & 760.619 & 18.114 & 3276.07 & 96.134 \\
\hline${ }^{230} \mathrm{Th}$ & 3722.82 & 250.653 & 6.732 & 1631.01 & 50.548 \\
\hline \multicolumn{6}{|l|}{$\begin{array}{c}{ }^{226} \mathrm{Ra} \\
\text { subseries }\end{array}$} \\
\hline${ }^{226} \mathrm{Ra}$ & 4102.93 & 20.252 & 0.493 & 6048.52 & 147.913 \\
\hline${ }^{214} \mathrm{~Pb}$ & 3322.8 & 36.215 & 1.089 & 3860.75 & 117.279 \\
\hline${ }^{214} \mathrm{Bi}$ & 3232.6 & 40.930 & 1.266 & 3299.06 & 103.322 \\
\hline${ }^{235} \mathrm{U}$ & 190.01 & 54.755 & 28.816 & 161.95 & 114.052 \\
\hline \multicolumn{6}{|l|}{${ }^{232}$ Th series } \\
\hline${ }^{228} \mathrm{Ac}$ & 73.94 & 7.366 & 9.962 & 18.54 & 35.036 \\
\hline${ }^{208} \mathrm{Tl}$ & 57.54 & 5.259 & 9.139 & 29.047 & 59.621 \\
\hline Average & 65.74 & 6.313 & 9.602 & 23.795 & 45.798 \\
\hline${ }^{40} \mathrm{~K}$ & 523.9 & 106.509 & 20.330 & 759.155 & 165.234 \\
\hline
\end{tabular}

Table (3) :Activity concentrations of radionuclides in original samples, residuals and leachates (pregnant solutions) for sample No. $2 \mathrm{~S}$ (Shale).

\begin{tabular}{|c|c|c|c|c|c|}
\hline Radionuclide & $\begin{array}{l}\text { Original } \\
(\mathrm{Bq} / \mathrm{Kg})\end{array}$ & $\begin{array}{l}\text { Solution } \\
\text { (Bq/l) }\end{array}$ & $\begin{array}{c}\text { Leachability } \\
(\%)\end{array}$ & $\begin{array}{l}\text { Residual } \\
(\mathrm{Bq} / \mathrm{Kg})\end{array}$ & $\begin{array}{c}\text { Summation } \\
\text { (Residual+Solution/ } \\
\text { Original }(\%)\end{array}$ \\
\hline \multicolumn{6}{|l|}{${ }^{238}$ Useries } \\
\hline${ }^{234} \mathrm{Th}$ & 6159.5 & 1996.8 & 32.418 & 7754.0 & 158.305 \\
\hline${ }^{234 \mathrm{~m}} \mathrm{~Pa}$ & 6483.8 & 1682.6 & 25.950 & 5735.7 & 114.412 \\
\hline Average & 6321.7 & 1839.7 & 29.101 & 6744.8 & 135.794 \\
\hline${ }^{234} \mathrm{U}$ & 6127.6 & 1327.4 & 21.662 & 6917.2 & 134.548 \\
\hline${ }^{230} \mathrm{Th}$ & 4746.4 & 591.4 & 12.459 & 10426 & 232.121 \\
\hline \multicolumn{6}{|l|}{$\begin{array}{c}{ }^{226} \mathrm{Ra} \\
\text { subseries }\end{array}$} \\
\hline${ }^{226} \mathrm{Ra}$ & 4841.9 & 25.9 & 0.534 & 7711.9 & 159.809 \\
\hline${ }^{214} \mathrm{~Pb}$ & 4279.4 & 17.8 & 0.4159 & 5536.3 & 129.786 \\
\hline${ }^{214} \mathrm{Bi}$ & 4107.1 & 29.20 & 0.7109 & 4945 & 121.112 \\
\hline${ }^{235} \mathrm{U}$ & 328.8 & 95.8 & 29.136 & 288.82 & 116.976 \\
\hline \multicolumn{6}{|l|}{$\begin{array}{l}{ }^{232} \mathrm{Th} \\
\text { series }\end{array}$} \\
\hline${ }^{228} \mathrm{Ac}$ & 55.99 & 6.2 & 11.073 & 91.99 & 175.370 \\
\hline${ }^{208} \mathrm{Tl}$ & 60.72 & 6.79 & 11.182 & 64.93 & 118.115 \\
\hline Average & 58.35 & 6.50 & 11.139 & 78.46 & 145.604 \\
\hline${ }^{40} \mathrm{~K}$ & 342.8 & 143.07 & 41.735 & 619.42 & 222.429 \\
\hline
\end{tabular}

Table (4) :Activity concentrations of radionuclides in original samples, residuals and leachates (pregnant solutions) for sample 3S (Siltstone).

\begin{tabular}{|c|c|c|c|c|c|}
\hline Radionuclide & $\begin{array}{l}\text { Original } \\
(\mathrm{Bq} / \mathrm{Kg})\end{array}$ & $\begin{array}{l}\text { Solution } \\
(\mathbf{B q} / \mathbf{l})\end{array}$ & $\begin{array}{c}\text { Leachability } \\
(\%)\end{array}$ & $\begin{array}{l}\text { Residual } \\
(\mathbf{B q} / \mathbf{K g})\end{array}$ & $\begin{array}{c}\text { Summation } \\
\text { (Residual+Solution)/ } \\
\text { Original }(\%)\end{array}$ \\
\hline \multicolumn{6}{|l|}{${ }^{238}$ Useries } \\
\hline${ }^{234} \mathrm{Th}$ & 2610.67 & 759.19 & 29.080 & 2394.32 & 120.793 \\
\hline${ }^{234 \mathrm{~m}} \mathrm{~Pa}$ & 2032.92 & 568.12 & 27.946 & 1656.06 & 109.408 \\
\hline Average & 2321.79 & 663.6 & 28.581 & 1656.06 & 99.908 \\
\hline${ }^{234} \mathrm{U}$ & 3946.52 & 513.14 & 13.002 & 1346.25 & 47.1146 \\
\hline${ }^{230} \mathrm{Th}$ & 2230.04 & 92.3 & 4.138 & 2972.27 & 137.422 \\
\hline \multicolumn{6}{|l|}{$\begin{array}{c}{ }^{226} \mathrm{Ra} \\
\text { subseries }\end{array}$} \\
\hline${ }^{226} \mathrm{Ra}$ & 2385.84 & 15.5 & 0.649 & 2645.42 & 111.529 \\
\hline${ }^{214} \mathrm{~Pb}$ & 2099.3 & 8.6 & 0.409 & 2008.26 & 96.073 \\
\hline${ }^{214} \mathrm{Bi}$ & 2001.7 & 2.79 & 0.139 & 1807.02 & 90.413 \\
\hline${ }^{235} \mathrm{U}$ & 114.81 & 28.58 & 24.893 & 94.68 & 107.359 \\
\hline \multicolumn{6}{|l|}{${ }^{232} \mathrm{Th}$ series } \\
\hline${ }^{228} \mathrm{Ac}$ & 67.86 & 1.42 & 2.092 & 116.16 & 173.269 \\
\hline${ }^{208} \mathrm{Tl}$ & 68.55 & 5.12 & 7.469 & 81.67 & 126.608 \\
\hline Average & 68.2 & 3.27 & 4.794 & 98.91 & 149.83 \\
\hline${ }^{40} \mathrm{~K}$ & 562.4 & 92.12 & 16.379 & 906.37 & 177.540 \\
\hline
\end{tabular}

Table (5): Activity concentrations of radionuclides in original samples, residuals and leachates (pregnant solutions) for Wadi Um Hamd sample No. A1.

\begin{tabular}{|c|c|c|c|c|c|}
\hline Radionuclide & $\begin{array}{l}\text { Original } \\
(\mathbf{B q} / \mathbf{K g})\end{array}$ & $\begin{array}{c}\text { Solution } \\
(\mathrm{Bq} / \mathrm{l})\end{array}$ & $\begin{array}{c}\text { Leachability } \\
(\%)\end{array}$ & $\begin{array}{c}\text { Residual } \\
(\mathrm{Bq} / \mathbf{K g})\end{array}$ & $\begin{array}{c}\text { Summation } \\
\text { (Residual+Solution)/ } \\
\text { Original }(\%)\end{array}$ \\
\hline \multicolumn{6}{|l|}{${ }^{238}$ Useries } \\
\hline${ }^{234} \mathrm{Th}$ & 8670.9 & 4880.5 & 56.28 & 8630.8 & 155.823 \\
\hline${ }^{234 \mathrm{~m}} \mathrm{~Pa}$ & 8580.8 & 4700.8 & 54.78 & 8650.9 & 155.599 \\
\hline Average & 8625.85 & 4790.65 & 55.53 & 8640.85 & 155.712 \\
\hline${ }^{234} \mathrm{U}$ & 8540.71 & 7620.8 & 89.22 & 9962.7 & 205.87 \\
\hline${ }^{230} \mathrm{Th}$ & 8625.89 & 3441.9 & 39.90 & 12140.72 & 180.64 \\
\hline \multicolumn{6}{|l|}{$\begin{array}{c}{ }^{226} \mathrm{Ra} \\
\text { subseries }\end{array}$} \\
\hline${ }^{226} \mathrm{Ra}$ & 10015.5 & 750.7 & 7.49 & 20440.9 & 211.58 \\
\hline${ }^{214} \mathrm{~Pb}$ & 8910.9 & 165.91 & 1.86 & 19655.8 & 222.44 \\
\hline${ }^{214} \mathrm{Bi}$ & 8890.1 & 160.82 & 1.80 & 18140.7 & 205.86 \\
\hline${ }^{235} \mathrm{U}$ & 400.10 & 210.8 & 52.68 & 330.9 & 135.39 \\
\hline \multicolumn{6}{|l|}{${ }^{232} \mathrm{Th}$ series } \\
\hline${ }^{228} \mathrm{Ac}$ & 60.7 & 6.1 & 10.05 & 65.4 & 117.79 \\
\hline${ }^{208} \mathrm{Tl}$ & 58.9 & 5.9 & 10.016 & 60.1 & 112.05 \\
\hline Average & 59.8 & 6 & 10.033 & 62.75 & 114.92 \\
\hline${ }^{40} \mathrm{~K}$ & 815.7 & 90.9 & 11.14 & 1194.2 & 157.54 \\
\hline
\end{tabular}


Table (6): Activity concentrations of radionuclides in original samples, residuals and leachates (pregnant solutions) for Wadi Um Hamd sample No. A2.

\begin{tabular}{|c|c|c|c|c|c|}
\hline Radionuclide & $\begin{array}{l}\text { Original } \\
(\mathbf{B q} / \mathbf{K g})\end{array}$ & $\begin{array}{c}\text { Solution } \\
(\mathbf{B q} \mathbf{l} \mathbf{l})\end{array}$ & $\begin{array}{c}\text { Leachability } \\
(\%)\end{array}$ & $\begin{array}{c}\text { Residual } \\
\text { (Bq/Kg) }\end{array}$ & $\begin{array}{c}\text { Summation } \\
\text { (Residual+Solution)/ } \\
\text { Original }(\%)\end{array}$ \\
\hline \multicolumn{6}{|l|}{${ }^{238}$ Useries } \\
\hline${ }^{234} \mathrm{Th}$ & 8690.9 & 4885.9 & 56.22 & 8840.12 & 157.935 \\
\hline${ }^{234 \mathrm{~m}} \mathrm{~Pa}$ & 8595.8 & 4710.8 & 54.80 & 8885.55 & 158.174 \\
\hline Average & 8643.35 & 4798.35 & 55.51 & 8862.83 & 158.054 \\
\hline${ }^{234} \mathrm{U}$ & 8570.12 & 7635.9 & 89.09 & 9980.80 & 205.55 \\
\hline${ }^{230} \mathrm{Th}$ & 8655.52 & 3455.1 & 39.91 & 12160.32 & 180.406 \\
\hline \multicolumn{6}{|l|}{$\begin{array}{c}{ }^{226} \mathrm{Ra} \\
\text { subseries }\end{array}$} \\
\hline${ }^{226} \mathrm{Ra}$ & 10050.52 & 775.32 & 7.714 & 20455.82 & 211.244 \\
\hline${ }^{214} \mathrm{~Pb}$ & 8950.18 & 185.21 & 2.069 & 19675.21 & 221.899 \\
\hline${ }^{214} \mathrm{Bi}$ & 8899.13 & 185.81 & 2.087 & 18165.15 & 206.209 \\
\hline${ }^{235} \mathrm{U}$ & 410.58 & 230.12 & 56.047 & 340.21 & 138.90 \\
\hline \multicolumn{6}{|l|}{${ }^{232}$ Th-series } \\
\hline${ }^{228} \mathrm{Ac}$ & 62.91 & 6.12 & 9.728 & 70.22 & 121.34 \\
\hline${ }^{208} \mathrm{Tl}$ & 60.89 & 5.51 & 9.049 & 66.81 & 118.771 \\
\hline Average & 61.9 & 5.81 & 9.388 & 68.515 & 120.055 \\
\hline${ }^{40} \mathrm{~K}$ & 825.12 & 98.35 & 11.919 & 1210.1 & 158.576 \\
\hline
\end{tabular}

Table (7): Activity concentrations of radionuclides in original samples, residuals and leachates (pregnant solutions) for Wadi Sad EI Banat Sample No. A3.

\begin{tabular}{|c|c|c|c|c|c|}
\hline Radionuclide & $\begin{array}{c}\text { Original } \\
(\mathrm{Bq} / \mathrm{Kg})\end{array}$ & $\begin{array}{c}\text { Solution } \\
(\mathbf{B q} / \mathbf{l})\end{array}$ & $\begin{array}{c}\text { Leachability } \\
(\%)\end{array}$ & $\begin{array}{c}\text { Residual } \\
(\mathbf{B q} / \mathbf{K g})\end{array}$ & $\begin{array}{c}\text { Summation } \\
\text { (Residual+Solution)/ } \\
\text { Original }(\%)\end{array}$ \\
\hline \multicolumn{6}{|l|}{${ }^{238}$ Useries } \\
\hline${ }^{234} \mathrm{Th}$ & 1060.8 & 51.4 & 4.84 & 1660.3 & 161.35 \\
\hline${ }^{234 \mathrm{~m}} \mathrm{~Pa}$ & 1015.7 & 50.8 & 5.00 & 1401.7 & 143.00 \\
\hline Average & 1038.25 & 51.1 & 4.92 & 1531 & 152.17 \\
\hline${ }^{234} \mathrm{U}$ & 1230.8 & 82.5 & 6.70 & 3060.9 & 255.40 \\
\hline${ }^{230} \mathrm{Th}$ & 991.5 & 25.4 & 2.56 & 1681.81 & 172.18 \\
\hline \multicolumn{6}{|l|}{$\begin{array}{c}{ }^{226} \mathrm{Ra} \\
\text { subseries }\end{array}$} \\
\hline${ }^{226} \mathrm{Ra}$ & 1210.61 & 10.12 & 0.835 & 1715.81 & 172.18 \\
\hline${ }^{214} \mathrm{~Pb}$ & 1092.6 & 8.70 & 0.796 & 1518.2 & 139.74 \\
\hline${ }^{214} \mathrm{Bi}$ & 1078.2 & 10.89 & 1.00 & 1382.7 & 129.24 \\
\hline${ }^{235} \mathrm{U}$ & 45.73 & 2.4 & 5.24 & 70.8 & 166.42 \\
\hline \multicolumn{6}{|l|}{${ }^{232} \mathrm{Th}$ series } \\
\hline${ }^{228} \mathrm{Ac}$ & 104.71 & 4.20 & 4.01 & 112.81 & 111.74 \\
\hline${ }^{208} \mathrm{Tl}$ & 103.30 & 5.10 & 4.93 & 114.8 & 116.06 \\
\hline Average & 104.00 & 4.65 & 4.47 & 113.80 & 113.90 \\
\hline${ }^{40} \mathrm{~K}$ & 181.21 & 80.82 & 44.60 & 322.31 & 222.96 \\
\hline
\end{tabular}

Table (8): Activity concentrations of radionuclides in original samples, residuals and leachates (pregnant solutions) Wadi Sad El Banat Sample No.A4.

\begin{tabular}{|c|c|c|c|c|c|}
\hline Radionuclide & $\begin{array}{l}\text { Original } \\
(\mathrm{Bq} / \mathrm{Kg})\end{array}$ & $\begin{array}{l}\text { Solution } \\
\text { (Bq/l) }\end{array}$ & $\begin{array}{c}\text { Leachability } \\
(\%)\end{array}$ & $\begin{array}{l}\text { Residual } \\
\text { (Bq/Kg) }\end{array}$ & $\begin{array}{c}\text { Summation } \\
\text { (Residual+Solution)/ } \\
\text { Original }(\%)\end{array}$ \\
\hline \multicolumn{6}{|l|}{${ }^{238}$ Useries } \\
\hline${ }^{234} \mathrm{Th}$ & 1070.51 & 60.21 & 5.62 & 1670.81 & 161.70 \\
\hline${ }^{234 \mathrm{~m}} \mathrm{~Pa}$ & 1030.10 & 61.35 & 5.95 & 1410.25 & 142.85 \\
\hline Average & 1050.30 & 60.78 & 5.78 & 1540.53 & 152.27 \\
\hline${ }^{234} \mathrm{U}$ & 1238.22 & 88.12 & 7.11 & 3080.53 & 255.9 \\
\hline${ }^{230} \mathrm{Th}$ & 998.85 & 37.10 & 3.71 & 1697.25 & 173.63 \\
\hline \multicolumn{6}{|l|}{$\begin{array}{c}{ }^{226} \mathrm{Ra} \\
\text { subseries }\end{array}$} \\
\hline${ }^{226} \mathrm{Ra}$ & 1220.15 & 19.18 & 1.57 & 1725.18 & 142.96 \\
\hline${ }^{214} \mathrm{~Pb}$ & 1098.12 & 12.15 & 1.10 & 1530.21 & 140.45 \\
\hline${ }^{214} \mathrm{Bi}$ & 1085.28 & 15.81 & 1.45 & 1395.55 & 130.04 \\
\hline${ }^{235} \mathrm{U}$ & 48.9 & 3.5 & 7.15 & 75.82 & 162.20 \\
\hline \multicolumn{6}{|l|}{${ }^{232} \mathrm{Th}$ series } \\
\hline${ }^{228} \mathrm{Ac}$ & 112.54 & 5.21 & 4.62 & 118.71 & 110.1 \\
\hline${ }^{208} \mathrm{Tl}$ & 105.92 & 5.42 & 5.09 & 116.82 & 115.3 \\
\hline Average & 109.23 & 5.31 & 4.85 & 117.76 & 112.7 \\
\hline${ }^{40} \mathrm{~K}$ & 187.58 & 85.55 & & 328.85 & 220.9 \\
\hline
\end{tabular}

Table (9): Activity concentrations of radionuclides in original samples, residuals and leachates (pregnant solutions) for granite sample No. 2G.

\begin{tabular}{cccccc}
\hline Radionuclide & Original & Solution & Leachability & Residual & $\begin{array}{c}\text { Summation } \\
\text { (Residual+solution)/ }\end{array}$ \\
$(\mathbf{B q} / \mathbf{K g})$ & $(\mathbf{B q} / \mathbf{l})$ & $(\%)$ & $(\mathbf{B q} / \mathbf{K g})$ & \begin{tabular}{c} 
Original $(\%)$ \\
\hline
\end{tabular}
\end{tabular}

\begin{tabular}{|c|c|c|c|c|c|}
\hline & & & & & \\
\hline \multicolumn{6}{|l|}{${ }^{238}$ Useries } \\
\hline${ }^{234} \mathrm{Th}$ & 2989.57 & 872.83 & 29.196 & 3076.61 & 132.107 \\
\hline${ }^{234 \mathrm{~m}} \mathrm{~Pa}$ & 2363.53 & 727.29 & 30.771 & 2543.2 & 138.373 \\
\hline Average & 2676.55 & 800.05 & 29.891 & 2809.9 & 134.87 \\
\hline${ }^{234} \mathrm{U}$ & 2397.13 & 1336.35 & 55.747 & 8155.31 & 395.959 \\
\hline${ }^{230} \mathrm{Th}$ & 2269.87 & 483.74 & 21.311 & 3870.3 & 191.819 \\
\hline \multicolumn{6}{|l|}{$\begin{array}{c}{ }^{226} \mathrm{Ra} \\
\text { subseries }\end{array}$} \\
\hline${ }^{226} \mathrm{Ra}$ & 2258.98 & 2.502 & 0.1107 & 4293.01 & 190.153 \\
\hline${ }^{214} \mathrm{~Pb}$ & 1925.9 & 17.90 & 0.926 & 3936.71 & 205.338 \\
\hline${ }^{214} \mathrm{Bi}$ & 1901.6 & 17.92 & 0.942 & 3651.32 & 192.955 \\
\hline${ }^{235} \mathrm{U}$ & 115.14 & 33.00 & 28.664 & 54.52 & 76.021 \\
\hline \multicolumn{6}{|c|}{${ }^{232} \mathrm{Th}$ series } \\
\hline${ }^{228} \mathrm{Ac}$ & 88.74 & 3.90 & 4.398 & 132.39 & 153.596 \\
\hline${ }^{208} \mathrm{Tl}$ & 74.58 & 12.71 & 17.043 & 56.72 & 93.106 \\
\hline Average & 801.66 & 8.30 & 10.172 & 94.56 & 125.971 \\
\hline${ }^{40} \mathrm{~K}$ & 1195 & 100.65 & 8.422 & 2096.44 & 183.857 \\
\hline
\end{tabular}


Table (10): Activity concentrations of radionuclides in original samples, residuals and leachates (pregnant solutions) for granite sample No. 5G.

\begin{tabular}{|c|c|c|c|c|c|}
\hline Radionuclide & $\begin{array}{l}\text { Original } \\
(\mathrm{Bq} / \mathrm{Kg})\end{array}$ & $\begin{array}{c}\text { Solution } \\
\text { (Bq/l) }\end{array}$ & $\begin{array}{c}\text { Leachability } \\
(\%)\end{array}$ & $\begin{array}{l}\text { Residual } \\
\text { (Bq/Kg) }\end{array}$ & $\begin{array}{c}\text { Summation } \\
\text { (Residual+solution)/ } \\
\text { Original }(\%)\end{array}$ \\
\hline \multicolumn{6}{|l|}{${ }^{238}$ Useries } \\
\hline${ }^{234} \mathrm{Th}$ & 2705.37 & 950.55 & 35.135 & 3076.77 & 148.863 \\
\hline${ }^{234 \mathrm{~m}} \mathrm{~Pa}$ & 2660.02 & 992.98 & 37.329 & 2749.6 & 140.697 \\
\hline Average & 2682.69 & 971.76 & 36.223 & 2913.18 & 144.815 \\
\hline${ }^{234} \mathrm{U}$ & 2958.02 & 1330.17 & 44.968 & 7016.07 & 282.156 \\
\hline${ }^{230} \mathrm{Th}$ & 2549.74 & 772.15 & 30.283 & 3906.2 & 183.483 \\
\hline \multicolumn{6}{|l|}{$\begin{array}{c}{ }^{226} \mathrm{Ra} \\
\text { subseries }\end{array}$} \\
\hline${ }^{226} \mathrm{Ra}$ & 2316.73 & 23.569 & 1.017 & 4100.64 & 178.018 \\
\hline${ }^{214} \mathrm{~Pb}$ & 2020.1 & 34.486 & 1.707 & 3868.76 & 193.220 \\
\hline${ }^{214} \mathrm{Bi}$ & 2016.2 & 47.268 & 2.344 & 3474.90 & 174.693 \\
\hline${ }^{235} \mathrm{U}$ & 123.7 & 42.455 & 34.320 & 56.20 & 79.754 \\
\hline \multicolumn{6}{|l|}{${ }^{232} \mathrm{Th}$ series } \\
\hline${ }^{228} \mathrm{Ac}$ & 95.14 & 13.411 & 14.096 & 112.24 & 132.072 \\
\hline${ }^{208} \mathrm{Tl}$ & 83.1 & 25.317 & 30.465 & 56.48 & 98.432 \\
\hline Average & 89.12 & 19.364 & 21.728 & 84.361 & 116.388 \\
\hline${ }^{40} \mathrm{~K}$ & 1264 & 189.480 & 14.990 & 189.48 & 180.540 \\
\hline
\end{tabular}

Table (11) represents the relation between the gamma attenuation and $(\mathrm{Pb})$ concentration. From the previously results, it can be concluded that $\mathrm{Pb}$ played its role as a good attenuated element for gamma radiation. The $\gamma$ attenuation increases with the increase in the $\mathrm{Pb}$ concentration in the measured sample.

Table (11): The relation between the gamma attenuation and (pb) concentration.

\begin{tabular}{|c|c|c|c|c|c|c|}
\hline $\begin{array}{c}\text { Sample } \\
\text { No. }\end{array}$ & $\begin{array}{l}{ }_{238}^{\text {Original- }} \\
(\mathbf{B q} / \mathbf{K g})\end{array}$ & $\begin{array}{l}{ }^{238} \mathbf{U} \\
(\mathbf{B q} / \mathbf{K g})\end{array}$ & $\begin{array}{l}\text { Solution- } \\
{ }^{238} \mathbf{U} \\
(\mathrm{Bq} / \mathrm{l})\end{array}$ & $\begin{array}{c}\text { Summation- } \\
\text { Original }\end{array}$ & $\begin{array}{l}\text { Attenuation } \\
\text { Factor }(\%)\end{array}$ & $\begin{array}{c}\mathrm{Pb} \\
\text { Concentration } \\
(\mathbf{p p m})\end{array}$ \\
\hline $2 \mathrm{~S}$ & 509.8 & 543.9 & 148.36 & 182.46 & 35.79 & 1865.28 \\
\hline $17 \mathrm{~S}$ & 230.88 & 273.0 & 101.49 & 143.61 & 62.2 & 1688.96 \\
\hline A1 & 695.63 & 696.84 & 386.34 & 387.55 & 55.71 & 2061.13 \\
\hline $\mathrm{A} 2$ & 697.04 & 714.74 & 386.96 & 404.66 & 58.05 & 2060.91 \\
\hline A3 & 83.73 & 123.47 & 4.12 & 43.86 & 52.38 & 3854.12 \\
\hline A4 & 84.70 & 124.24 & 4.9 & 44.44 & 52.47 & 3852.85 \\
\hline $2 \mathrm{G}$ & 190.60 & 226.6 & 64.52 & 100.52 & 52.74 & 1111.36 \\
\hline $5 \mathrm{G}$ & 216.35 & 234.93 & 78.37 & 96.95 & 44.81 & 1102.08 \\
\hline
\end{tabular}

The high leaching efficiency \% present in sample A1 where leachability for ${ }^{234} \mathrm{U}(89.22 \%)$ and for ${ }^{238} \mathrm{U}(55.53 \%) .{ }^{238} \mathrm{U}$ and ${ }^{234} \mathrm{U}$ have the same chemical behavior, So $33.69 \%$ of ${ }^{234} \mathrm{U}$ was transferred physically by $\alpha$ - recoil effect [14].

Also, in sample A2 ${ }^{234} \mathrm{U}$ have leachability (89.09\%) and ${ }^{238} \mathrm{U}$ have $(55.51 \%)$. So $(33.58 \%)$ of ${ }^{234} \mathrm{U}$ was transferred physically. The low leachability in samples $\mathrm{A} 3$ and $\mathrm{A} 4$ for ${ }^{238} \mathrm{U} 4.92 \%$ and $5.78 \%$ respectively. For ${ }^{234} \mathrm{U} 6.7 \%$ and $7.11 \%$ respectively. So A3 has physically transferred $1.78 \%$ and A4 has $1.33 \%$ physically transferred.

In $2 \mathrm{G}{ }^{234} \mathrm{U}$ has leachability $55.74 \%$ and ${ }^{238} \mathrm{U}$ has $29.89 \%$, so the physically transferred $22 \%$. In Sample $5 \mathrm{G}$ the leachability of ${ }^{234} \mathrm{U} 44.96 \%$ and for ${ }^{238} \mathrm{U} 36.22$, so the transferred physically by $\alpha$ - recoil $8.74 \%$.

From leaching studies have shown that isotopes are leached to the same extent but that is not observed in ${ }^{238} \mathrm{U}$, hence the difference between ${ }^{234} \mathrm{U}$ and ${ }^{238} \mathrm{U}$ leaching could represent the ${ }^{234} \mathrm{U} \alpha$ - recoil as shown in table (12).

Table (12): Chemical and physical transfer for ${ }^{234} U$ for studied sample.

\begin{tabular}{ccc}
\hline Sample No. & Chemical Transfer (\%) & Physical Transfer (\%) \\
\hline A1 & 55.53 & 33.69 \\
A2 & 55.51 & 33.58 \\
A3 & 4.92 & 1.78 \\
A4 & 5.78 & 1.33 \\
2G & 33.74 & \\
5G & 36.22 & 22 \\
\hline
\end{tabular}

\section{CONCLUSION}

It is noticed that, the activities of the residual are higher than the activity of the solution. There is difference in $\gamma$ - activity between the summation of activities of residual and solution with the activity of the original sample. The difference in $\gamma$ - activity is sometimes $-\mathrm{ve}$ and in other time $+\mathrm{ve}$, that its means summation is greater than the original. This phenomenon is due to the attenuation of the gamma activities during 
the leaching processes, for existence a high concentration of lead $(\mathrm{Pb})$. The leaching processes by using acid solution may have lead to clean the grain surface and permit the gamma activities of the inner grains to be measured. Also, the residual have high concentration of ${ }^{226} \mathrm{Ra}$ in all samples. The ${ }^{235} \mathrm{U}$ has the same leachability as ${ }^{238} \mathrm{U}$ but in leaching process find that ${ }^{234} \mathrm{U}$ has leachability higher than ${ }^{238} \mathrm{U}$ due to $\alpha$ recoil. So the leachability of ${ }^{234} U$ is divided into chemical transfer (\%) physical transfer (\%) due to $\alpha$ recoil.

\section{REFERENCES}

[1] E. Svoukis, and H. Testertos, "Indoor and outdoor in situ high -resolution gamma radiation measurements in urban areas of Cyprus, Radiat. Prot.Dosim., Vol.123, pp. 384-390, (2006).

[2] E. M. Ibrahim, "Behavior of Radionuclides in USeries During Processing of Uranium Bearing Rock Materials", Ph.D. Thesis, Faculty of Women for Art, Science and Education, Physics Department, Egypt, (2011).

[3] D.R. Metzler, (2004),Uranium Mining: Environmental Impact, Encyclopedia of Energy, Vol. 6, pp. 299-315, (2004).

[4] I.E. El Aassy, M.G. El Feky, M.A. El Kasaby, E. M. Ibrahim, S. Sewefi and R. M. Attia, "Behavior of Radionuclides during Acidic Leaching Processes of Different Rock Materials, Allouga Locality, Southwestern Sinai, Egypt", International Journal of Scientific \& Engineering Research, V. 8, Issue 1, ISSN 2229-5518, (2017).

[5] I.E El Aassy, A.A. Nada, M.M. El Galy, M.G. El Feky, T.M. Abd El Maksoud, S.M. Talaat and E.M. Ibrahim, "Behavior and Environmental Impacts of Radionuclides during the Hydrometallurgy of Calcareous and Argillaceous Rocks, Southwestern Sinai, Egypt", Applied Radiation and Isotopes, Vol. 70, pp. 1024-1033, (2012).

[6] R. Tykva and D. Berg, "Man-Made and natural radioactivity in environmental pollution and radio chronology". Klwer Academic, P.O. Box 17, 3300 AA Dordecht, Netherlands, (2004).

[7] G. Gilmore and J.D. Hemingway, "Practical Gamma-Ray Spectrometry". John Wiley \& Sons, Chichester, (2008).

[8] IAEA, Preparation and certification of IAEA gamma spectrometry reference materials, RGU-1, RGTh-1 and RGK-1, Report-IAEA/RL/148, International Atomic Energy Agency, (1987).

[9] R.M. Anjos, R. Veiga, T. Soares, A.M.A. Santos, J.G. Aguia, Frasca' MHBO, D. Brage JAP, Uze^da, L. Mangia L., A. Facure, B. Mosquera, C. Carvalho , Gomes PRS, "Natural radionuclide distribution in Brazilian commercial granites", Radiat Meas Vol. 39, pp. 245-253, (1987).

[10] S. Turhan and L. Gu"ndu"z, "Determination of specific activity of ${ }^{226} \mathrm{Ra},{ }^{232} \mathrm{Th}$ and ${ }^{40} \mathrm{~K}$ for assessment of radiation hazards from Turkish pumice samples", J. Environ Radioact Vol. 99, pp. 332-342, (2008).

[11] R.N. Nair, F. Sunny and S.T. Manikandan, "Modelling of Decay Chain Transport in Groundwater from Uranium Tailings Ponds", Applied Mathematical Modelling, Vol. 34, pp. 2300-2311, (2010).

[12] K. Gravani, C. Papachristodoulou, A. Oikonomouand K. Ioannidies, "A study of Ancient Pottery by Means of X-ray Fluorescence, Multivariate Statics and Mineralogical Analysis", Analytic Chemical Acta, pp. 347-353, (2006).

[13] UNSCEAR, United Nations Scientific Committee on the Effect of Atomic Radiation, "Sources and Effects of Ionizing Radiation". Report to General Assembly with Scientific Annexes A and B, E. 10XI. 3., United Nations, New York, (2010).

[14] W. Jinlong, Du. Jinzhou and Bi. Qianqian, "Natural Radioactivity Assessment of Surface Sediments in the Yangtze Estuary", Marine Pollution Bulletin, Vol. 114, pp. 602-608, (2017). 